\title{
Numerical Study of Fluid Dynamics in Oscillating Deep Cavities
}

\author{
Indukuri Jagadeesh Varma ${ }^{1}$, Ranjith Maniyeri ${ }^{2}$, Sangmo Kang ${ }^{3}$ \\ ${ }^{1,2}$ (Department of Mechanical Engineering,National Institute of Technology Karnataka, Surathkal, Mangalore- \\ 575025, India) \\ ${ }^{3}$ (Department of Mechanical Engineering, Dong-A University, 840Hadan2Dong, Saha-Gu, Busan, Republic of \\ Korea)
}

\begin{abstract}
Study of lid driven cavity problem has long been considered as a testing for Navier-Stokes numerical solvers, and also these problems find more immense industrial applications like short dwell and flexible blade coaters. In this paper, we present a numerical model to study the deep oscillating lid driven cavity problem using staggered grid based finite volume method. In order to capture the fluid dynamic behavior inside the cavity, the continuity and momentum equations are solved, and then the simulations are performed for three different cases of wall oscillation with aspect ratio 2.0. First case is for single wall (top wall oscillation) and the second case is for parallel wall (top and bottom walls oscillation in same direction) and final one is antiparallel wall (top and bottom walls oscillation in opposite directions). In this work, the pressure Poisson equation is solved using successive over relaxation method, and fluid velocities are obtained using first order Euler explicit scheme. Firstly, the developed code in FORTRAN is validated by comparing our results with other researcher's results. Later, the numerical simulations are carried out for exploring the vortex behavior inside the oscillating deep cavity for a fixed Reynolds number for all the above mentioned three cases. It is observed that better mixing characteristics in a cavity can be achieved for low Reynolds number when compared to lid moving with finite velocity.
\end{abstract}

Keywords: Lid driven cavity, Finite volume method, Oscillating wall, Navier-Stokes equations, Staggered grid.

\section{Introduction}

Two dimensional study of lid-driven cavity has been studied over years due to its simple geometry and also this problem remains as a bench mark for the identification of complex flow behaviors. Mostly this problem finds more industrial applications like short dwell, spray coatings etc., and there are many a number of simulation works were reported on single-wall and two-sided-wall motion of lid-driven square cavity and bifurcations using different numerical methods [1-9]. From the literature, it is found that very less amount of works have been reported on oscillating lid driven square cavity [10,11]. The present numerical model is based on staggered grid system using finite volume method, which is not much reported in the literature. Inspired by this, the present paper aims to develop a computational model to investigate the flow in a oscillating deep liddriven square cavity using staggered grid based finite volume method.Firstly, the developed code will be validated using bench mark results of Ghia et al. [9]. Secondly, numerical simulations will be performed to observe the vortex behavior in oscillating lid-driven square cavity for fixed Reynolds number $(\mathrm{Re}=100)$ with one sided and two-sided (parallel and anti-parallel wall) lid motions.

\section{Mathamatical Modelling And Numerical Procedure}

Fig. 1 shows the schematic diagram of the physical domain representing single sided oscillating liddriven square cavity with top wall oscillating with varying velocity as a cosine function i.e., $U \cos (\omega t)$, where $\omega$ is oscillating frequency and $t$ is time period. The continuity and Navier-Stokes equationsare usedto study the fluid motion in a lid-driven square cavity which are shown below

$\nabla \cdot \mathbf{u}=0$

$\rho\left(\frac{\partial \mathbf{u}}{\partial t}+\mathbf{u} . \nabla \mathbf{u}\right)=-\nabla \mathrm{p}+\mu \nabla^{2} \mathbf{u}$

whereu is fluid velocity, $\rho$ is fluid density, $p$ is fluid pressure, $\mu$ is dynamic viscosity of the fluid.The continuity and Navier-Stokes equations are non-dimensionalised,using characteristic length $\left(\mathrm{L}_{\mathrm{c}}\right)$ and characteristic velocity (U)which are shown in eqns. (3) and (4).

$\nabla \cdot \mathbf{u}=0$

$\left(\frac{\partial \mathbf{u}}{\partial \mathrm{t}}+\mathbf{u} \cdot \nabla \mathbf{u}\right)=-\nabla \mathrm{p}+\frac{1}{\mathrm{Re}} \nabla^{2} \mathbf{u}$ 
where Re is Reynolds number and given by, $\operatorname{Re}=\frac{\rho \mathrm{UL} c}{\mu}$

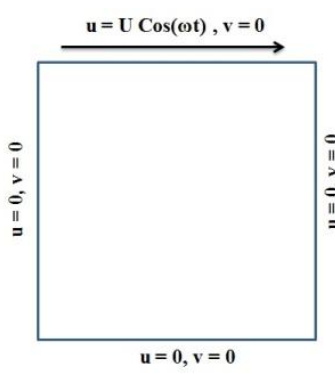

Fig. 1. Schematic diagram of the physical domain representing oscillating lid-driven square cavity.

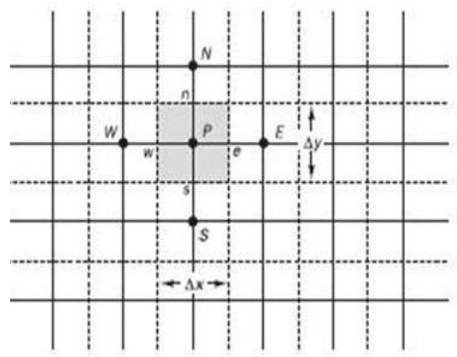

Fig. 2. Staggered grid system

Finite volume method represents small control volume surrounding each point on mesh nodes. In this method, computational domain is divided in to elementary volumes and integration is performed at each elementary volumes. The main advantage of finite volume method is that these methods are conservative in nature compared to finite difference method performing discretization at only one single node.

In the present work, staggered grid based finite volume method is used for discretization of continuity and momentum equations.In figure 2, a general node on staggered grid system is represented as (i,j). The velocity in $\mathrm{x}$-direction is defined at nodes $\mathrm{w}$ and $\mathrm{e}$ as $\mathrm{u}_{\mathrm{i}-1, \mathrm{j}}$ and $\mathrm{u}_{\mathrm{i}, \mathrm{j}}$. Similarly, velocity in y-direction is defined at the nodes $s$ and $n$ as $v_{i, j-1}$ and $v_{i, j}$. The pressure is defined at the center of grid as $p_{i, j}$ at the point $P$.

Here in this numerical strategy, the pressure Poisson equation is solved using successive over-relaxation method and first-order Euler-explicit method is used for time advancement to obtain the fluid velocities.

\section{Results And Discussion}

Numerical simulations are performed by developing a code in FORTRAN. Firstly, the developed code is used to study the fluid flow behavior in a lid-driven square cavity for $\mathrm{Re}=100$ with only top wall moving with constant dimensionless velocity $\mathrm{U}=1$. Fig. 3shows the comparison of steady state $\mathrm{x}$-component velocity along the vertical centerline in the cavity at $\mathrm{Re}=100$ between present and Ghia et al. [9] results. The agreement between present results and those of Ghia et al. [9] is excellent.

In this paper, we represent a deep cavity with aspect ratio 2.0 with finite wall motion at $\mathrm{Re}=100$ which is shown in Fig. 4. Figure 4 shows the formation of primaryand secondary vortex in the cavity. It can be seen that the primary vortex formation is due to the lid motion and it is penetrated only up to depth 1.0. It is due to the presence of adverse pressure which acts on the fluid, where as the secondary vortex which moves in counterwise of primary is due to the pressure and shear forces acting on the secondary vortex.

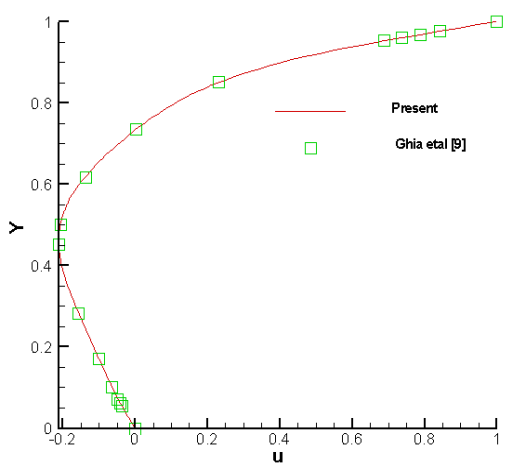

Fig. 3. Variation of $\mathrm{u}$ velocity along $\mathrm{y}$-direction at $\mathrm{Re}=100$

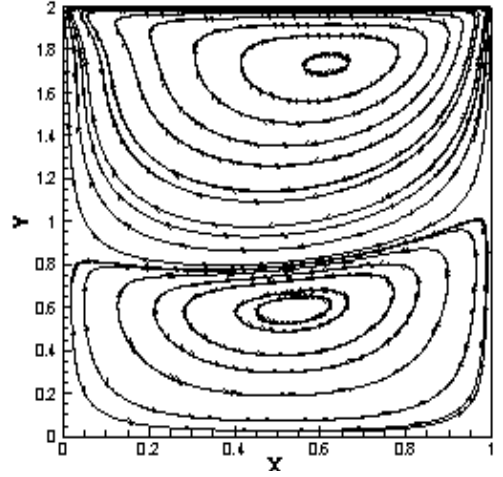

Fig. 4. Streamline plot at $\mathrm{Re}=100$ 


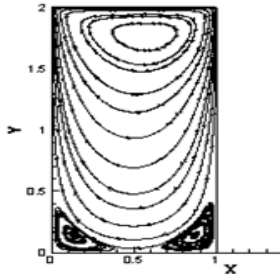

$\mathrm{t}=0.2 \mathrm{~T}, \mathrm{u} / \mathrm{U}=0.30901$

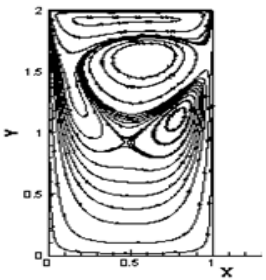

$\mathrm{t}=0.35 \mathrm{~T}, \mathrm{u} / \mathrm{U}=-0.5877$

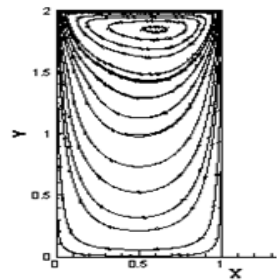

$\mathrm{t}=\mathrm{T}, \mathrm{u} / \mathrm{U}=1$

Fig.5. One sided wall motion with cosine variation for $\mathrm{t}=0.2 \mathrm{~T}$ to $\mathrm{t}=\mathrm{T}$ and amplitude $=0.5$

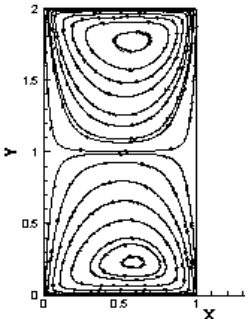

$\mathrm{t}=0.2 \mathrm{~T}, \mathrm{u} / \mathrm{U}=0.30901$

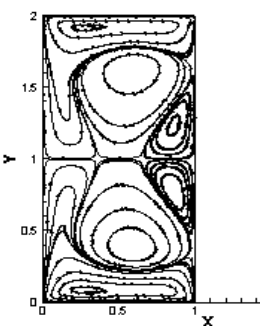

$\mathrm{t}=0.35 \mathrm{~T}, \mathrm{u} / \mathrm{U}=-0.5877$

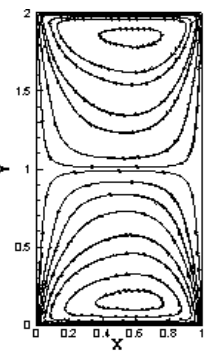

$\mathrm{t}=\mathrm{T}, \mathrm{u} / \mathrm{U}=1$

Fig.6. Two sided wall motion (parallel wall) with cosine variation for $\mathrm{t}=0.2 \mathrm{~T}$ to $\mathrm{t}=\mathrm{T}$ and amplitude $=0.5$

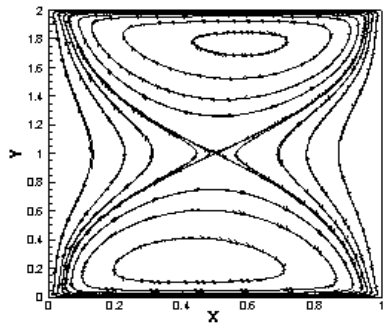

$\mathrm{t}=0.2 \mathrm{~T}, \mathrm{u} / \mathrm{U}=0.30901$

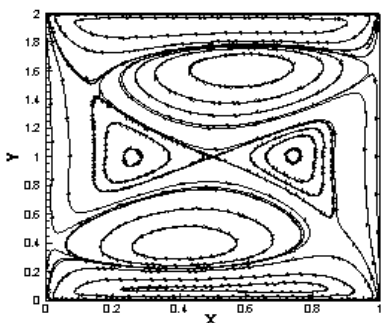

$\mathrm{t}=0.35 \mathrm{~T}, \mathrm{u} / \mathrm{U}=-0.5877$

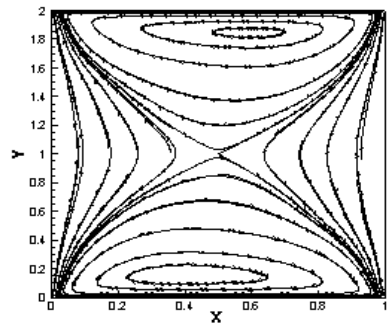

$\mathrm{t}=\mathrm{T}, \mathrm{u} / \mathrm{U}=1$

Fig.7. Two sided wall motion (anti-parallel wall) with cosine variation for $\mathrm{t}=0.2 \mathrm{~T}$ to $\mathrm{t}=\mathrm{T}$ and amplitude $=0.5$

From Fig. 5 it is noticed that at $t=0.2 \mathrm{~T}$, the entire cavity is dominated by primary vortex moving in clock-wise direction and also it can be observed at corner small secondary vortices moving in counterclock-wise sense. At time $\mathrm{t}=0.35 \mathrm{~T}$ the lid changes its direction due to this a secondary counter clock-wise rotating vortex can be observed near the top wall along with the primary vortex which is already formed before, which stops the penetration of the secondary vortex. As the time progresses counterclock-wise rotating vortex at the top of the cavity increases its size and primary vortex size keeps on declining. At the end of the first half time, that is up to $t=0.5 \mathrm{~T}$, the counter clock-wise rotating secondary vortices keeps on increasing its mass by mixing with the primary and corner vortices. Further in second half time i.e. $t=0.7 \mathrm{~T}$ to $\mathrm{T}$, the formation of vortices is reversal of the first half time.

Further, numerical simulations are done for the case of parallel wall motion with same cosine variation for amplitude $=0.5$ and $\omega=\frac{2 \pi}{6}$ for fixed Re=100. Fig. 6 shows the streamline patterns for the case of parallel wall motion. From Fig. 6 it is observed that, at time $t=0.2 \mathrm{~T}$ streamline patterns with two rotating primary vortices symmetrical to each other are formed from the centerline of $y$ axis, which shows the similar behavior for the case of parallel wall motion with finite velocity. As the time advances $(t=0.35 \mathrm{~T})$ the secondary vortices startsdeveloping at the top and bottom of the cavity due to the negative motion of lid as shown and the primary vortices which are already present starts reducing its size. Finally at the end of first half time $(t=0.5 \mathrm{~T})$ the entire cavity is dominated by secondary vortices by mixing with the primary vortices, and similarly the second half time $(\mathrm{t}=0.7 \mathrm{~T}$ to $\mathrm{t}=\mathrm{T})$, the formation of primary and secondary vortices is reversal to that of first half cycle $(\mathrm{t}=0.2 \mathrm{~T}$ to $\mathrm{t}=0.5 \mathrm{~T})$. 
Finally, the simulations are done for the case of anti-parallel wall motion with cosine variation for amplitude $=0.5$ and $\omega=\frac{2 \pi}{6}$ for fixed $\mathrm{Re}=100$. Fig. 7 shows the streamline patterns for the case of anti-parallel wall motion. From the Fig. 7 at time $t=0.2 \mathrm{~T}$ to $\mathrm{t}=\mathrm{T}$, it can be seen that the streamline patterns with primary vortices are formed at geometric center and as the time progresses $(t=0.35 \mathrm{~T})$ the secondary vortices starts developing at the top and bottom of the cavity due to the negative motion of the lid followed by reducing the size of primary vortices. At the end of first half time $(\mathrm{t}=0.5 \mathrm{~T})$ the entire cavity is dominated by secondary vortices by mixing with primary vortices, and at time $\mathrm{t}=0.7 \mathrm{~T}$ to $\mathrm{t}=\mathrm{T}$ the formation primary and secondary vortices is reversal that of the first half.

\section{Conclusions}

In this paper, using a staggered grid based finite volume method a numerical study is performed to capture the fluid dynamics features of an oscillating lid-driven square cavity. First of all, the numerical code is validated by comparing our numerical results with that of the other researcher's results. Later, the flow is investigated for single wall motion with oscillating lid deep cavity. It is found that initially primary vortices occupy entire cavity and then slowly development of secondary vortices occur and entire cavity is occupied by the secondary vortices for first half time. In the second half time, the same pattern occurs in reversal manner due to lid changing its direction for fixed Reynolds number. Then, from the flow analysis for parallel wall motion it is observed that the streamline patterns with two rotating primary vortices symmetrical to each other are formed from the centerline of $y$ axis. As the time progresses the development of secondary vortices at the top and bottom of the cavity can be observed and the primary vortices reduce its size. At the end of first half time, the entire cavity is dominated by secondary vortices and also a well mixing of fluid can be observed. Finally, from the flow inspected for anti-parallel wall motion it is found that the streamline patterns with primary vortices can be observed at initial time. As the time progresses the development of secondary vortices at the top and bottom of the cavity can be seen and the primary vortices reduce its size similar to that of the previous cases. Finally, we conclude that better mixing can happen for low Reynolds number using oscillation of wall motion.

[1]. Perumal D A and Dass A K 2010 Simulation of incompressible flows in two-sided lid-driven square cavities Part II LBM CFD Letters 2(1) 25-38.

[2]. Lemee T, G. Kasperski, G. Labrosse, R. Narayanan, Multiple stable solutions in the 2D symmetrical two-sided square lid-driven cavity,Computers \& Fluids 119 (2015) 204-212.

[3]. Swapan Pandit K, Jatin C Kalita, D C Dalal, A transient higher order compact scheme for incompressible viscous flows on geometries beyond rectangular,Journal of Computational Physics 225(2007) 1100-1124.

[4]. Patil D V, K N Lakshmisha, B Rogg, Lattice Boltzmann simulation of lid-driven flow in deep cavities, Computers \& fluids 35 (2006) 1116-1125.

[5]. Li-Song Lin, Hung-Wen Chang, Chao-An Lin,Multi relaxation time lattice Boltzmann simulations of transition in deep 2D lid-driven cavity using GPU, Computers \& fluids 80 (2013) 381-387.

[6]. Cheng M and Hung K C, Vortex structure of steady flow in a rectangular cavity,Computers \& Fluids 35 (2006) 1046-1062.

[7]. Zdanski P S B, M.A Ortega, Nide G.C.R Fico Jr, Numerical study of the flow over shallow cavities, Computers \& Fluids 32 (2003) 953-974.

[8]. Wahba E M, Multiplicity of states for two-sided and four-sided lid-driven cavity flows, Computers \& Fluids 38 (2008) 247-253.

[9]. Ghia U,K.N Ghia, C.T Shin, High-Re solutions for incompressible flow using the Navier- Stokes equations and a multigrid method,Journal of Computational Physics 48 (1983) 387-411.

[10]. Siva Subrahmanyam Mendu, P K Das, Fluid flow in a cavity driven by an oscillating lid - A simulation by lattice Boltzmann method,Journal of Mechanics B/Fluids 39(2013) 59-70.

[11]. Zhenhong Hu, Xing Zheng, Qing-Wei Ma, Wen-Yang Duan, Fluid flow in a cavity driven by an oscillating lid by an improved incompressible SPH,Procedia Engineering 126 (2015) 275 - 279. 